\title{
Utilizing an abstraction relation document in grey-box testing approach
}

\begin{abstract}
This paper describes the test oracle generation from an abstraction relation document that is documented using Parnasôs Module Documentation (MD) method. This work is part of ongoing research that addresses the problem of improving the effectiveness of fault detection. We focus our work on unit/module testing where each module may consist of several programs. The aim of our project is to investigate the strategies and techniques to automate module testing. In particular, we investigate the use of MD that is written in standard mathematical notation to automate the process of test oracle generation and test execution.
\end{abstract}

Keyword: Specification-based testing; Test oracle; Automated module testing 